\title{
Metástases Vertebrais: Breve Revisão do Estado da Arte
}

\section{Spinal Metastasis: Brief State of the Art Review}

\author{
Bruno Santiago ${ }^{1,2,3}$, João Levy Melancia ${ }^{1}$, Manuel Cunha e Sá1,2,3,4
}

Autor Correspondente: Bruno Santiago [bruno.santiago@jmellosaude.pt] Travessa do Castro N³, 1350-070 Lisboa, Portugal

\section{RESUMO}

O tratamento das metástases vertebrais sofreu uma evolução relevante nos últimos anos, com o desenvolvimento tecnológico da radioterapia/radiocirurgia estereotáxica, novas combinações de quimioterapia e imunoterapia, assentes numa nova subclassificação genética de alguns tumores, permitindo planos terapêuticos mais personalizados e em fases mais precoce no curso da doença. A cirurgia mantém um papel incontornável no tratamento da dor, instabilidade mecânica e deterioração neurológica causadas pela infiltração tumoral. A necessidade de uma citorredução menos agressiva a par da utilização de técnicas cirúrgicas menos invasivas, permitem uma recuperação mais rápida e com menor morbilidade.

A avaliação diagnóstica e orientação terapêutica da doença metastática vertebral devem, à semelhança de tantos outros domínios da medicina e em especial da oncologia, estar subordinadas a uma abordagem e decisão multidisciplinares. O objetivo é o de individualizar o plano de tratamento que melhor sirva os interesses específicos de cada doente, tendo em conta as múltiplas opções disponíveis.

PALAVRAS-CHAVE: Metástase Neoplásica; Neoplasias da Coluna Vertebral/cirurgia; Neoplasias da Coluna Verte$\mathrm{bral} /$ radioterapia

\section{ABSTRACT}

The treatment of spinal metastasis has undergone a significant evolution in recent years, with the technological development of stereotactic radiosurgery, new combinations of chemotherapy and immunotherapy, based on a new genetic subclassification of some tumors, allowing more personalized therapeutic plans and at an earlier stage in the course of the disease. Surgery plays an essential role in the treatment of pain, mechanical instability and neurological deterioration caused by tumor infiltration. The need for a less aggressive cytoreduction coupled with the use of less invasive surgical techniques allows a faster recovery and lower morbidity.

1. Neurocirurgia, Hospital CUF Infante Santo, Cluster Tejo, Lisboa Portugal. 2. Neurocirurgia, Clínica CUF Almada, Almada, Portugal. 3. Serviço de Neurocirurgia, Hospital Garcia de Orta, EPE, Almada, Portugal. 4. Neurocirurgia, Hospital CUF Cascais, Cascais, Portugal.

Recebido: 20/08/2017 - Aceite: 04/09/2017 
The diagnostic evaluation and therapeutic orientation of spinal metastatic disease should, like in many other fields of medicine and in particular of oncology, be subject to a multidisciplinary approach and decision. The objective is to individualize the treatment plan that best serves the specific interests of each patient, taking into account the multiple options available.

KEYWORDS: Neoplasm Metastasis; Spinal Neoplasms/radiotherapy; Spinal Neoplasms/surgery

\section{INTRODUÇÃO}

Os avanços recentes no tratamento da doença oncológica, nomeadamente com a introdução recente de novos fármacos de quimioterapia e de imunoterapia, têm melhorado os resultados terapêuticos e a esperança de vida, o que obriga a uma análise e compreensão robustas dos fatores que influenciam a duração e qualidade de vida dos doentes. ${ }^{1} \bigcirc$ osso é o terceiro órgão mais afetado por metástases e cerca de 50\% destas são na coluna vertebral. ${ }^{1,2}$ Alguns estudos sugerem que pelo menos 30\% dos doentes oncológicos terão metástases vertebrais no curso da doença. ${ }^{2}$ Os tumores primários mais frequentes são os da mama, próstata e pulmão seguidos dos tumores de células renais, melanoma, tiroide e colorretal, bem como neoplasias hematológicas. ${ }^{2,3}$ De todos os tumores, o mieloma múltiplo é o que tem maior tendência de metastização no ráquis. A fase de maior incidência é entre os 40 e os 65 anos de idade, o que corresponde ao período de maior risco de doença oncológica. A metastização vertebral pode constituir a manifestação inicial da doença neoplásica numa percentagem que pode ir até $10 \%$ dos doentes oncológicos. ${ }^{2}$ O envolvimento torácico é o mais frequente (70\%), seguido da coluna lombar (20\%), coluna cervical e sacro. ${ }^{3,4}$ A grande maioria são osteolíticas, embora os tumores da próstata e mama possam originar lesões osteoblásticas. São quase exclusivamente extradurais (95\%) e muito raramente intradurais (5\%) ou intramedulares. ${ }^{2}$

A compressão da medula espinhal estima-se que ocorra em 20\% dos doentes e pode ter um enorme impacto na capacidade funcional e qualidade de vida dos doentes. ${ }^{2-4}$

Os doentes com metastização do ráquis representam um grupo muito heterogéneo, tendo em conta que a sobrevida depende da histologia do tumor primário, do grau de doença sistémica e da presença de co-morbilidades. O facto de que, em média, apenas 10\% a 20\% dos doentes estarão vivos aos dois anos após o diagnóstico, deve ser tomado em conta aquando do processo de tomada de decisão sobre o tratamento. ${ }^{2}$

tratamento das metástases vertebrais beneficiou muito com os enormes avanços técnicos registados nas últimas décadas, nomeadamente com o desenvolvimento de técnicas cirúrgicas menos invasivas e pela implementação da radioterapia/radiocirurgia estereotáxica conformacional, que melhorou significativamente as taxas de controlo local, mesmo nos tumores tipicamente radiorresistentes.

\section{AVALIAÇÃO DO DOENTE E DECISÃO DE TRATAMENTO 1. DISCUSSÃO MULTIDISCIPLINAR}

Um dos mais importantes desenvolvimentos no tratamento das metástases da coluna vertebral é a compreensão generalizada de que o processo de decisão deve ser coletivo, para garantir a melhor forma de orientação de um doente específico com as opções disponíveis no armamentário terapêutico, permitindo assim cumprir cinco objetivos principais ${ }^{1,2}$ :

1. Controlar a doença local

2. Tratar a dor, seja esta por fratura patológica ou efeito expansivo do tumor

3. Manter a estabilidade da coluna vertebral

4. Manter ou melhorar a função neurológica

5. Melhorar a qualidade de vida

O consenso terapêutico deve ser multidisciplinar e obtido a partir de reuniões que congreguem os oncologistas médicos, radioterapeutas, neurorradiologistas, anatomopatologista, fisiatra, enfermeiro de oncologia e psicólogo. O neurocirurgião de coluna tem aqui um papel relevante e praticamente único na avaliação dos fatores de instabilidade mecânica e de compressão neurológica e na implementação de medidas imediatas para a sua resolução. A discussão multidisciplinar pode ser facilitada utilizando sistemas de consulta "virtual" ou com recurso a novas tecnologias de comunicação e partilha de imagem eletrónica. ${ }^{1}$

\section{ALGORITMOS DE TRATAMENTO}

Para facilitar o processo de decisão terapêutica foram descritas várias escalas centradas na síntese da estimativa de sobrevida e decisão de propor ou não tratamento cirúrgico (e de que tipo). As mais conhecidas são as de Tomita ${ }^{5}$ e a de Tokuhashi, ${ }^{6}$ que foram desenvolvidas antes do advento da radiocirurgia estereotáxica.

Mais recentemente surgiram vários algoritmos de orientação clínica que incluem todas as alternativas terapêu- 
ticas. A primeira data de 2008 e decorre da enorme experiência do grupo de Boriani et al no tratamento da patologia tumoral primária e secundária da coluna vertebral.3,7 Mais recentemente foi descrito o algoritmo $\mathrm{NOMS}^{2,8}$ (N - neurological; $\mathrm{O}$ - oncological; $\mathbf{M}$ - mechanical; $\mathbf{S}$ - systemic disease) utilizado nos últimos 15 anos no Hospital Memorial Sloan Kettering em Nova Iorque e que inclui a radiocirurgia estereotáxica no paradigma de tratamento. Em 2015 o Metastatic Spine Disease Multidisciplinary Working Group publicou também um algoritmo de orientação clínica. Este tem por base recomendações para cinco grupos de doentes: assintomáticos, com dor, mas sem fratura, fratura estável, fratura instável e compressão medular epidural. ${ }^{9}$

O algoritmo NOMS tem sido agregador de maior consenso, por ser adaptável à introdução de inovações terapêuticas, apresentar uma forma muito sistematizada de avaliação e por incluir outras escalas de decisão (instabilidade mecânica e estimativa da sobrevida e prognóstico, por exemplo).

\subsection{Algoritmo de tratamento NOMS}

\section{$N$ - Avaliação Neurológica}

A avaliação neurológica centra-se, em primeiro lugar, na presença ou não de défice motor ou sensitivo dos membros por mielopatia ou radiculopatia compressivas, obrigando a uma avaliação neurocirúrgica urgente e forte consideração para cirurgia descompressiva. Por outro lado, é fundamental a avaliação radiológica da doença metastática epidural e do grau de compressão medular, mesmo na ausência de defeito neurológico. Neste âmbito, o Spine Oncology Study Group (SOSG) propôs e validou uma escala (Tabela 1) de 6 pontos, tendo por base imagens de Ressonância magnética nas ponderações T2 axiais de ressonância magnética, para classificar o grau de compressão medular. ${ }^{10} \mathrm{Na}$ ausência de instabilida-

TABELA 1. Escala de classificação do grau de compressão medular ${ }^{10}$ - Spinal Oncology Study Group.

\begin{tabular}{c|c} 
Grau & Sem compressão do espaço subaracnoideu \\
\hline $1 a$ & $\begin{array}{l}\text { Obliteração parcial do espaço subaracnoideu } \\
\text { sem deformação do saco tecal }\end{array}$ \\
1 1b & $\begin{array}{l}\text { Deformação do saco tecal sem contacto com } \\
\text { a medula }\end{array}$ \\
1 1c & $\begin{array}{l}\text { Deformação do saco tecal com contacto } \\
\text { medular, sem compressão }\end{array}$ \\
2 & $\begin{array}{l}\text { Compressão medular, mas com liquor visível } \\
\text { ao redor da medula }\end{array}$ \\
3 & $\begin{array}{l}\text { Obliteração completa do espaço } \\
\text { subaracnoideu com compressão medular }\end{array}$
\end{tabular}

de e défice neurológico os graus 0, 1 a e 1b podem ser considerados para radiação com tratamento inicial, sobretudo nos tumores muito radiossensíveis. No grau 10 deve ser considerado o tratamento cirúrgico seguido de radiocirurgia. Doentes com compressão medular grau 2 e 3 requerem tratamento cirúrgico urgente. 2,8,10

No caso dos tumores hematológicos muito radiossensíveis - plasmocitoma/mieloma e linfoma - alguns autores têm recomendado a radioterapia convencional ou radiocirurgia, caso seja viável em tempo útil, combinada com corticoides em alta dose, mesmo em doentes com défice neurológico e independentemente do grau de compressão medular, com resposta clínica e imagiológica muito favorável. ${ }^{11}$

\section{O - Avaliação Oncológica}

No essencial, a avaliação oncológica consiste na previsão de resposta do tumor aos tratamentos farmacológicos e de radiação atualmente disponíveis. No caso das metástases vertebrais o tratamento mais eficaz e menos invasivo para o controlo local da doença é a radioterapia, embora a quimioterapia possa ser considerada terapêutica de primeira linha em casos selecionados, nomeadamente nos neuroblastomas, tumores de células germinais, linfomas e sarcomas de Ewing. ${ }^{3}$ A análise genética dos tumores revolucionou o tratamento médico, ${ }^{1}$ em particular nos carcinomas da mama, certos tumores pulmonares, melanoma e linfoma anaplásico, em que a pesquisa de mutações específicas permite prever uma melhor resposta à quimioterapia ou imunoterapia. ${ }^{1} \bigcirc$ uso de agentes anti-VEGF (vascular endotelial growth factor) aumentou muito a sobrevida sem progressão de vários tumores sólidos, sobretudo nas lesões hipervascularizadas como é o caso do tumor de células renais. ${ }^{12}$

Os corticoides são usados para controlo da dor em complemento aos analgésicos e na presença de compressão medular. ${ }^{2}$ Podem ainda ter um efeito oncolítico, por exemplo no mieloma múltiplo, linfoma e timoma. ${ }^{4}$ Os bifosfonatos ou o denosumab já se demonstrou terem efeito na redução da incidência de fraturas em doentes com metástases da mama ou mieloma múltiplo. ${ }^{4}$ Os tumores da mama e próstata podem ser muito sensíveis à hormonoterapia. ${ }^{3}$

No que diz respeito aos tratamentos com radiação externa, os tumores são classificados como radiossensíveis ou radiorresistentes consoante a sua resposta à radioterapia convencional (sem técnicas conformacionais). ${ }^{2-4}$ Os tumores muito radiossensíveis são o mieloma múltiplo, seminoma e o linfoma. Os tumores sólidos, por outro lado, exibem um largo espectro de resposta à radiação. Classificados como radiossensíveis ou de sensibilidade 

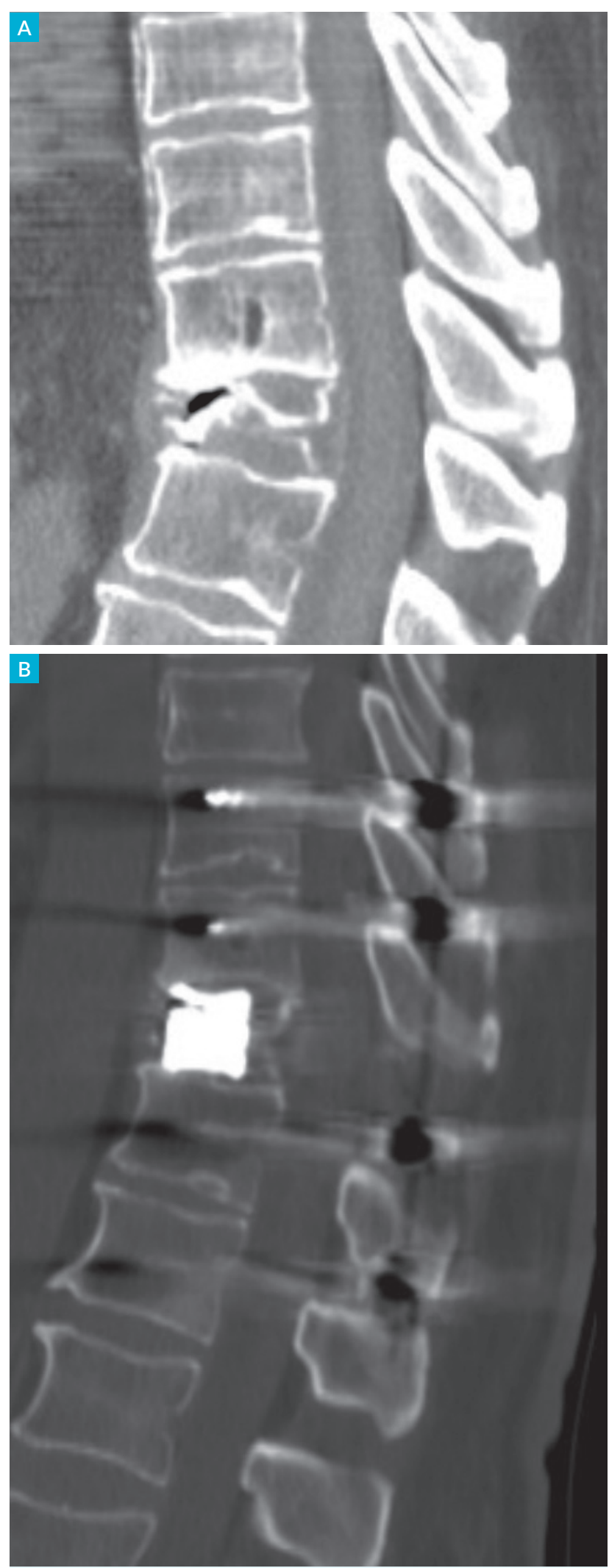

FIGURA 1. A instabilidade mecânica é uma indicação cirúrgica independentemente do controlo oncológico.

A: Tomografia computorizada (TC) pré-operatória (corte sagital) com grave fratura somática de D11 e cifose segmentar (pontuação SINS de 13), num doente de 51 anos com plasmacitoma previamente tratado com radioterapia/quimioterapia e com dor axial mecânica severa.

B: TC pós-operatório (corte sagital), mostrando corpectomia de D11, reconstrução da coluna anterior e fixação posterior nos dois níveis adjacentes, por abordagem exclusivamente posterior. Anatomia patológica sem evidência de recidiva tumoral. intermédia à radiação são os tumores da mama, próstata, ovário e carcinomas neuro-endócrinos. Os tumores de células renais, tiroide, hepatocelular, cólon, sarcomas e pulmão de não pequenas células são considerados radiorresistentes. $^{4}$

O uso de tecnologia guiada por imagem tem permitido a administração de doses altas de radiação com elevada precisão espacial (IGRT - image guided radiation therapy), mantendo dentro dos limites de segurança a exposição da medula e outras estruturas vitais (esófago, plexos cervical e lombar, por exemplo).2,4 A radiocirurgia estereotáxica consiste na administração de altas doses de radiação muito focalizada, com precisão (utilizando as plataformas de IGRT) e pode ser administrada numa sessão única, tipicamente 16 a 24 Gy, ou num esquema hipofracionado (3 - 5 frações) de 24 a 30 Gray (Gy). A capacidade de tratar estas lesões com doses letais de radiação conformacional, melhorou as taxas de controlo local de 30\% aos três meses com radioterapia convencional (por exemplo 30 Gy em 10 frações), para mais de 90\% quando usada como terapêutica definitiva ou adjuvante pós-operatória, ${ }^{2}$ incluindo tumores tipicamente radiorresistentes. A radioterapia estereotáxica tem sido utilizada com tratamento primário, adjuvante pós-operatório e como retratamento em doentes com progressão da doença local.1-4 A sua utilização é ideal e de primeira linha em doentes com metástases pequenas para evitar a progressão para compressão medular ou instabilidade mecânica. Também pode ser utilizada paliativamente na doença terminal para controlo da dor, com uma eficácia de 80\%-85\%. Os doentes tratados com estas técnicas de alta dose têm um risco associado de fratura vertebral pós-tratamento entre 11\% e 39\%, ${ }^{4}$ pelo que se devem considerar estratégias de vigilância destes doentes e, em casos selecionados, ponderar vertebroplastia profilática (envolvimento $>50 \%$ do corpo vertebral ou atingimento dos elementos posteriores). ${ }^{13}$

Resumindo, os tumores radiossensíveis podem ser tratados com radioterapia convencional ou radiocirurgia, embora esta última tenha vindo a ser utilizada de forma crescente em doentes oligometastáticos para maior eficácia. Os tumores radiorresistentes requerem radiocirurgia para controlo local duradouro, 2,8 no entanto, nos centros que não têm esta modalidade disponível deve ser considerado o tratamento cirúrgico antes da radioterapia convencional

\section{M - Instabilidade Mecânica}

A instabilidade mecânica representa por si só uma indicação formal para estabilização cirúrgica, independentemente do grau de compressão medular ou radiossensibilidade do tumor$^{8}$ (Fig. 1). Tem sido definida como "a perda 
de integridade da coluna vertebral quando submetida a cargas fisiológicas, associada a dor relacionada com o movimento, deformidade progressiva ou sintomática e/ ou compromisso neurológico" (SOSG). ${ }^{8}$

A instabilidade pode ser avaliada do ponto de vista clínico e radiológico. Clinicamente acompanha-se de dor axial que agrava em carga i.e sentado, em pé ou com flexão do tronco e que alivia, pelo menos parcialmente, em decúbito. Distingue-se da dor por expansão tumoral por esta ser predominantemente noturna, independente da posição e com boa resposta aos corticoides e radioterapia. Em termos radiológicos é avaliada pela presença de fratura do corpo vertebral, envolvimento das facetas articulares e movimento anormal ou deformidade na radiografia dinâmica da coluna ou na comparação da radiografia estática em decúbito e em carga.

Em 2013 foi proposta uma escala de avaliação da estabilidade da coluna vertebral em doentes com tumores ver-

TABELA 2. SINS: Spinal Instability Neoplastic Score..$^{14-16}$

\begin{tabular}{|c|c|}
\hline & Pontuação \\
\hline \multicolumn{2}{|l|}{ Localização } \\
\hline $\begin{array}{l}\text { Juncional (occipital-C2; C7-T2; } \\
\text { T11-L1, L5-S1) }\end{array}$ & 3 \\
\hline Coluna móvel (C3-C6, L2-L4) & 2 \\
\hline Coluna semi-rígida (T3-T10) & 1 \\
\hline \multicolumn{2}{|l|}{ Coluna rígida (S2-S5) } \\
\hline \multicolumn{2}{|l|}{ Dor } \\
\hline Sim & 3 \\
\hline Ocasional, mas não mecânica & 1 \\
\hline Sem dor & 0 \\
\hline \multicolumn{2}{|l|}{ Lesão Óssea } \\
\hline Lítica & 2 \\
\hline Mista (lítica/blástica) & 1 \\
\hline Blástica & 0 \\
\hline \multicolumn{2}{|l|}{ Alinhamento radiológico } \\
\hline Subluxação/translação & 4 \\
\hline Deformidade de novo (cifose/escoliose) & 2 \\
\hline Alinhamento normal & 0 \\
\hline \multicolumn{2}{|l|}{ Colapso do corpo vertebral } \\
\hline$>50 \%$ & 3 \\
\hline$<50 \%$ & 2 \\
\hline $\begin{array}{l}\text { Sem colapso, mas com envolvimento > } 50 \% \\
\text { corpo vertebral }\end{array}$ & 2 \\
\hline Nenhum dos anteriores & 0 \\
\hline \multicolumn{2}{|l|}{ Envolvimento dos elementos posteriores } \\
\hline Bilateral & 3 \\
\hline Unilateral & 1 \\
\hline Nenhum dos anteriores & 0 \\
\hline \multicolumn{2}{|l|}{ Pontuação total } \\
\hline Estável & $0-6$ \\
\hline Indeterminado & $7-12$ \\
\hline Instável & $13-18$ \\
\hline
\end{tabular}

tebrais, o SINS - spinal instability neoplastic score (Tabela 2). ${ }^{14-16}$ Trata-se de uma escala com 18 pontos e inclui 6 parâmetros, a saber: localização, dor, alinhamento, grau de osteólise, colapso do corpo vertebral e envolvimento dos elementos posteriores. Lesões com uma pontuação baixa, entre 0 e 6, são geralmente estáveis e não requerem tratamento cirúrgico. Na presença de uma pontuação entre 13 e 18, é necessário o tratamento cirúrgico para restaurar a estabilidade da coluna vertebral. Lesões com um SINS intermédio, entre 7 e 13, podem necessitar de tratamento cirúrgico, numa avaliação caso-a-caso e devem ser sempre referenciados a uma opinião cirúrgica. ${ }^{8,13}$

Embora a sua utilidade prognóstica não esteja estabelecida, é uma ferramenta válida e muito útil na comunicação e referenciação entre oncologistas, radioterapeutas, fisiatras e cirurgiões.

\section{S - Avaliação Sistémica}

Todas as decisões de tratamento dependem da previsão da capacidade fisiológica do doente tolerar a intervenção proposta, assim como da avaliação da extensão da doença sistémica e "carga" tumoral.2,3,8 A presença de comorbilidades, a idade do doente, tratamentos prévios e a histologia do tumor influenciam decisivamente essa análise. É da maior relevância nos doentes considerados para tratamento cirúrgico e obriga a uma estratificação do risco e a um estadiamento preciso da doença, assentes num conhecimento da biologia e comportamento do tumor. Como regra geral, se existir quimioterapia disponível para o pós-operatório e a progressão sistémica não aparente ser suficientemente rápida para impedir a recuperação da intervenção, com uma estimativa de sobrevida mínima de 3 a 6 meses, os doentes devem ser considerados para cirurgia, 2,3,8 caso se determine estar indicada. Tem sido demonstrado que para determinados tipos histológicos de tumores, o envolvimento da coluna vertebral indica uma sobrevida muito curta, por exemplo no tumor do pulmão de não pequenas células, carcinoma do cólon e carcinoma de origem indeterminada, com sobrevidas médias de quatro meses após o tratamento cirúrgico. Pelo contrário, outros tumores estão associados a sobrevidas bem mais longas, o que faz com que o envolvimento oligometastático do ráquis, na presença de doença sistémica controlada, possa recomendar uma cirurgia mais agressiva do ponto de vista da ressecção oncológica.

Foram propostas várias escalas preditivas de sobrevida, sendo a mais fiável a escala de Tokuhashi modificada17 (Tabela 3), que se alicerça em seis parâmetros: condição geral (classificação de Karnofsky), número de metástases vertebrais, ósseas e em órgãos major, tumor primário 
e estado neurológico. Se o score total for $\leq 5$ a sobrevida estimada é inferior a três meses e apenas se recomenda tratamento paliativo. A sobrevida estimada é inferior a seis meses quando o score é $\leq 8$, enquanto que na presença de um valor $\geq 12$ a estimativa de sobrevida é superior a 12 meses. Mais recentemente foram descritas outras escalas como o Bauer modified score e o prognostic index for spinal metastases (PRISM). ${ }^{18}$

Vários autores têm destacado que o principal fator que contribui para a sobrevida é o número de metástases nos órgãos principais, independentemente da origem do tumor primário. Rades et al18 numa avaliação retrospetiva de 552 doentes com tumores sólidos com compressão medular epidural, tratados com radioterapia (30 Gy em 10 sessões), documentaram uma sobrevida aos seis meses de 88\%, 55\%, 13\% e 12\%, em doentes com envolvimento de 0, 1, 2, 3 e $\geq 4$ órgãos extrarraquidianos envolvidos, respetivamente. ${ }^{19}$

TABELA 3. Escala de Tokuhashi modificada. ${ }^{17}$

\begin{tabular}{|c|c|}
\hline Parâmetros & Pontuação \\
\hline \multicolumn{2}{|l|}{ Condição geral (Karnofsky) } \\
\hline Muito incapacitado (Karnofsky 10\%-40\%) & 0 \\
\hline Moderada (Karnofsky 50\%-70\%) & 1 \\
\hline Boa (Karnofsky 80\%-100\%) & 2 \\
\hline \multicolumn{2}{|l|}{$\mathrm{N}^{\circ}$ metástases ósseas extrarraquidianas } \\
\hline$\geq 3$ & 0 \\
\hline $1-2$ & 1 \\
\hline 0 & 2 \\
\hline \multicolumn{2}{|l|}{$\mathrm{N}^{\circ}$ metástases corpos vertebrais } \\
\hline$\geq 3$ & 0 \\
\hline 2 & 1 \\
\hline 1 & 2 \\
\hline \multicolumn{2}{|l|}{ Metástases em órgãos major } \\
\hline Irressecáveis & 0 \\
\hline Ressecáveis & 1 \\
\hline Sem metástases & 2 \\
\hline \multicolumn{2}{|l|}{ Tumor primário } \\
\hline Pâncreas, esófago, osteossarcoma, pulmão & 0 \\
\hline Fígado, vesícula, primário oculto & 1 \\
\hline Outros & 2 \\
\hline Rim, útero & 3 \\
\hline Reto & 4 \\
\hline Tiroide, próstata, mama & 5 \\
\hline \multicolumn{2}{|l|}{ Défice neurológico } \\
\hline Completo & 2 \\
\hline Incompleto & 1 \\
\hline Ausente & 0 \\
\hline Pontuação total & Sobrevida \\
\hline $0-8$ & $<6$ meses \\
\hline $9-12$ & $6-12$ meses \\
\hline $12-15$ & $>12$ meses \\
\hline
\end{tabular}

\section{AVANÇOS NO TRATAMENTO CIRÚRGICO}

\subsection{Vertebroplastia/cifoplastia}

A vertebroplastia é uma técnica que consiste na injeção de cimento ósseo de polimetilmetacrilato (PMMA), por via percutânea minimamente invasiva. ${ }^{13} \mathrm{Na}$ cifoplastia colocam-se balões expansíveis no corpo vertebral previamente à injeção de cimento, criando uma cavidade no corpo da vértebra, reduzindo, em situações específicas, o risco de extravasamento do material injetado e aumentando a capacidade de correção da deformidade vertebral existente em consequência da fratura.

É útil na estabilização de fraturas patológicas com dor axial mecânica, sem doença epidural ou compressão medular (Fig. 2), ou nas já referidas situações em que se preveja um risco aumentado de fratura após a radiocirurgia., 1,4,13 É uma técnica com um risco baixo de complicações, no entanto, o risco de extravasamento de cimento com défice neurológico de novo, é maior comparativamente às fraturas osteoporóticas, podendo atingir os 5\%.2,4,13,20 Isto deve-se à natureza da lesão, que muitas vezes compromete a cortical posterior do corpo vertebral. ${ }^{20}$

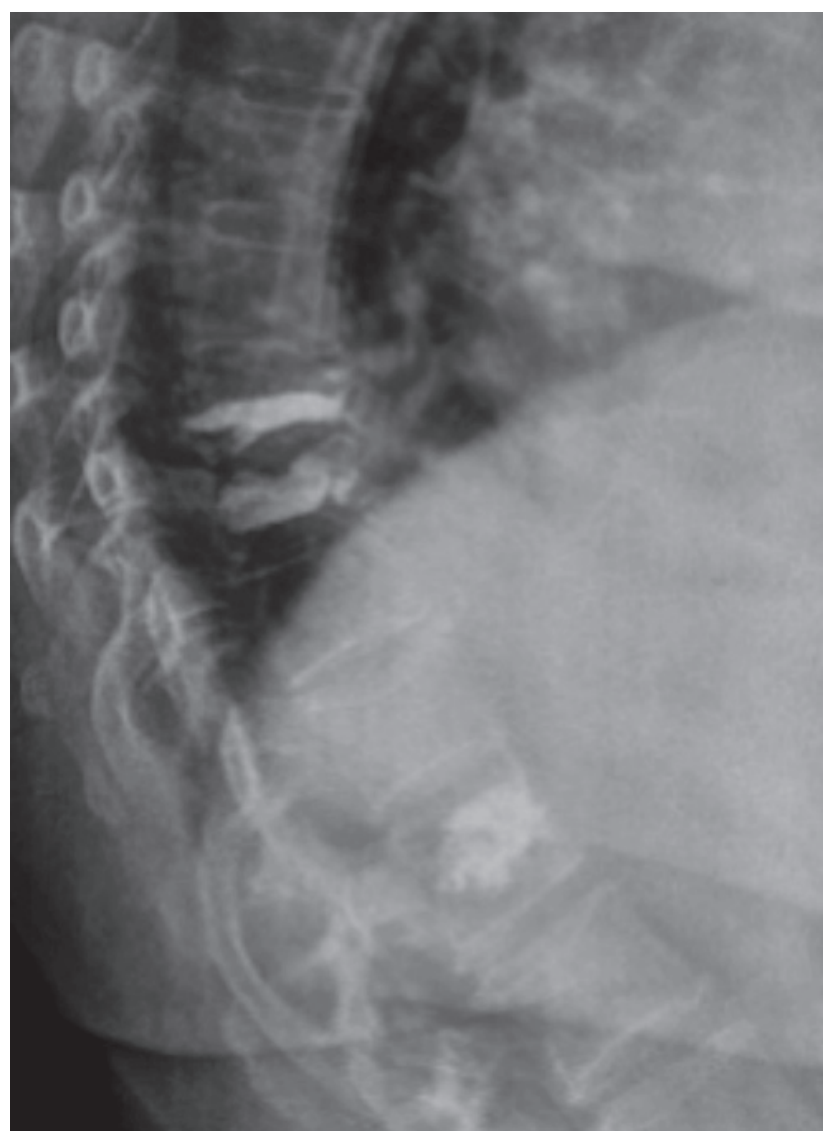

FIGURA 2. Doente de 60 anos com envolvimento metastático difuso da coluna vertebral (tumor primário da mama). Submetida a vertebroplastia nos 3 níveis em que apresentava fratura somática (D8, D9, D12), antes dos tratamentos de radioterapia, por dor axial mecânica. 


\subsection{Técnicas minimamente invasivas}

Para além da vertebroplastia, os avanços na instrumentação cirúrgica permitiram que as técnicas de fixação transpedicular percutâneas e afastadores tubulares sofresse um incremento nos últimos anos, permitindo descomprimir e estabilizar a coluna vertebral através de pequenas incisões e com menores percas hemáticas e menor risco infecioso, conduzindo a redução significativa da morbilidade perioperatória e a uma mais rápida e melhor recuperação pós-operatória. ${ }^{1}$ Não existe ainda nenhum estudo que valide a superioridade destas técnicas nos resultados a longo prazo, comparativamente à cirurgia aberta convencional, embora conceptualmente as vantagens sejam evidentes, desde que se consigam os mesmos objetivos em termos de estratégia cirúrgica. 1,2,21

\subsection{Cirurgia de separação}

A laminectomia simples, sem estabilização, deixou de ser a cirurgia de eleição na patologia metastática da coluna, não só pelo seu potencial de instabilização da coluna, mas também por claramente não permitir resolver de forma eficaz a compressão ventral ou deformidade raquidiana frequentemente presentes. ${ }^{2}$

Por outro lado, com o advento da radioterapia estereotáxica e radiocirurgia tornou-se menos necessário recorrer a cirurgias extensas de citorredução, com maior morbilidade cirúrgica.

Recentemente foi descrita a "cirurgia de separação", 2,8,21,22 que consiste na descompressão posterior clássica complementada com fixação posterior transpedicular nos dois níveis adjacentes e com a remoção adicional do segmento posterior no corpo vertebral do tumor, junto à medula, permitindo restaurar o espaço de liquor pré-medular, sem reconstrução da coluna anterior ou remoção exaustiva da massa tumoral no corpo vertebral ou paravertebral. Obtém-se assim uma margem segura para a aplicação de radiação citotóxica dentro dos limites de tolerabilidade da medula. Está indicada como tratamento de primeira linha complementada com radiocirurgia nos doentes com tumores radiorresistentes e compressão medular de alto grau. Moulding et al22 reportaram uma taxa de falência a um ano de apenas 6,3\% com a combinação da cirurgia de separação e radiocirurgia estereotáxica de 18-24 Gy numa única sessão. Numa série de 186 doentes Laufer et $a^{23}$ documentaram uma taxa de recorrência a um ano de 16,4\%. No entanto, essa taxa foi menor nos doentes com protocolo hipofracionado de alta dose de 24 a 30 Gy em 3 frações (4,1\%), ou sessão única de 24 Gy, (9\%). No grupo que foi submetido a um esquema de dose menor, 30 Gy em cinco sessões, a taxa de falência foi superior, atingindo os 22,6\%. A cirur- gia de separação pode ser combinada com a vertebroplastia para estabilização adicional da coluna anterior, se necessário for.

\subsection{Remoção oncológica "em bloco"}

Com o maior controlo local que a radiocirurgia oferece, a cirurgia oncológica com remoção em bloco total ou parcial da vértebra, tem sido cada vez menos recomendada. ${ }^{2}$ Embora se acompanhe de um maior risco de morbilidade, tem uma enorme eficácia em termos de controlo tumoral, com uma taxa de recorrência muitíssimo baixa. ${ }^{4,23} \mathrm{Em}$ doentes selecionados com doença sistémica controlada, que se apresentem com metástase única e histologia tipicamente radiorresistente, pode ser uma opção a considerar, sobretudo em centros que só têm radioterapia convencional disponível. Deve ainda ser considerada em doentes com progressão local de tumores previamente irradiados, em que a biologia do tumor é conhecida e favorável em termos de sobrevida. ${ }^{24}$

\subsection{Técnicas de ablação}

A ablação tumoral com radiofrequência é executada com a mesma técnica cirúrgica da vertebroplastia e pode ser efetuada no mesmo tempo, com o objetivo de destruir o tecido tumoral no corpo vertebral. ${ }^{1}$ Está contraindicada perante a existência de tecido tumoral a uma distância inferior a $1 \mathrm{~cm}$ do limite anterior da face ventral da medula, por risco de lesão medular iatrogénica. É necessária a monitorização da temperatura extradural e irrigação com soro fisiológico para reduzir a transmissão térmica. ${ }^{1}$ Os resultados a longo prazo não são claros, mas pode potencialmente ser utilizado em doentes com metástases osteolíticas irressecáveis, que não tolerem procedimentos cirúrgicos prolongados ou quando as opções convencionais de radioterapia ou radiocirurgia não se afigurem eficazes. ${ }^{1,9}$

\section{CONCLUSÃO}

O diagnóstico e tratamento das metástases vertebrais requer uma abordagem multidisciplinar, centrada no doente, em que as características específicas individuais no que diz respeito aos sintomas de dor e/ou défice neurológico, estado funcional, capacidade física, tratamentos prévios, probabilidade de complicações e prognóstico global sejam avaliadas conjuntamente. É fundamental utilizar algoritmos de orientação clínica, escalas de prognóstico e de risco de instabilidade mecânica, que facilitem o processo de decisão e para que os doentes beneficiem de todas as alternativas terapêuticas. As estratégias de tratamento tornaram-se mais sofisticadas e com opções menos invasivas, afetando positivamente a qualidade de vida e controlo local da doença. $\bigcirc$ trata- 
mento cirúrgico mantém o seu papel de destaque nos doentes com compressão medular, instabilidade mecânica, fratura do corpo vertebral e em caso de falência ou progressão local após tratamento com radioterapia/ radiocirurgia.

CONFLITOS DE INTERESSE: Os autores declaram não ter qualquer conflito de interesse na realização do presente trabalho.

FONTES DE FINANCIAMENTO: Não houve qualquer fonte de financiamento na realização do presente trabalho.

CONFIDENCIALIDADE DOS DADOS: Os autores declaram ter seguido os protocolos da sua instituição acerca da publicação dos dados de doentes.

PROTEÇÃO DE PESSOAS E ANIMAIS: Os autores declaram que os procedimentos seguidos na elaboração do presente trabalho estão em conformidade com as normas das comissões de investigação clínica e de ética, bem como da declaração de Helsínquia e da Associação Médica Mundial.

CONFLICTS OF INTEREST: The authors declare that they have no conflicts of interest.

FINANCIAL SUPPORT: This work has not received any contribution, grant or scholarship.

CONFIDENTIALITY OF DATA: The authors declare that they have followed the protocols of their work ceter on the publication of data from patients.

PROTECTION OF HUMAN AND ANIMAL SUBJECTS: The authors declare that the procedures followed were in accordance with the regulations of the relevant clinical research ethics committee and with those of the Code of Ethics of the World Medical Association (Declaration of Helsinki).

\section{REFERÊNCIAS}

1. Choi D, Bilsky M, Fehlings M, Fisher C, Gokaslan Z. Spine oncology-metastatic spine tumors. Neurosurgery. 2017;80: S131-7.

2. Joaquim AF, Powers A, Laufer I, Bilsky MH. An update in the management of spinal metastases. Arq Neuropsiquiatr. 2015;73:795-802.

3. Yan ML, Dirks MS, Tatsui CE, Rhines LD. Evaluation and decision making for metastatic spinal tumors. In: Vialle LR, editor. AO Spine Masters Series Metastatic Spinal Tumors. Berlin: Thieme; 2015. p.1-13.

4. Nater A, Fehlings MG. Assessment and Treatment of Metastatic Spinal Lesions. $7^{\text {th }}$ ed. In: Youmans \& Winn Neurological Surgery. Amsterdam: Elsevier; 2017. p. 2435-46.

5. Tomita K, Kawahara N, Kobayashi T, Yoshida A, Murakami $\mathrm{H}$, Akamaru T. Surgical strategy for spinal metastases. Spine. 2001;26:298-306.

6. Tokuhashi Y, Matsuzaki H, Toriyama S, Kawano H, Ohsaka S. Scoring system for the preoperative evaluation of metastatic spine tumor prognosis. Spine. 1990;15:1110-3.
7. Gasbarrini A, Li H, Cappuccio M, Mirabile L, Paderni S, Terzi $\mathrm{S}$, et al. Efficacy evaluation of a new treatment algorithm for spinal metastases. Spine. 2010;35:1466-70.

8. Laufer I, Rubin DG, Lis E, Cox BW, Stubblefield MD, Yamada Y, et al. The NOMS Framework: Approach to the treatment of spinal metastatic tumors. Oncologist. 2013;18:744-751.

9. Wallace AN, Robinson CG, Meyer J, Tran ND, Gangi A, Callstrom MR, et al. The Metastatic Spine Disease Multidisciplinary Working Group Algorithms. Oncologist. 2015;20:1205-15.

10. Bilsky MH, Laufer I, Fourney DR, Groff M, Schmidt MH, Varga PP, et al. Reliability analysis of the epidural spinal cord compression scale. J Neurosurg Spine. 2010;13:324-8.

11. Rades D, Conde-Moreno AJ, Cacicedo J, Segedin B, Rudat $\checkmark$, Schild SE. Excellent outcomes after radiotherapy alone for malignant spinal cord compression from myeloma. Radiol Oncol. 2016;50:337-40.

12. Gregory TM, Coriat R, Mir O. Prognostic scoring systems for spinal metastases in the era of anti-VEGF therapies. Spine. 2013;38:965-6.

13. Georgy BA. Vertebroplasty technique in metastatic disease. Neuroimaging Clin N Am. 2010;20:169-77.

14. Fisher CG, DiPaola CP, Ryken TC, Bilsky MH, Shaffrey CI, Berven $\mathrm{SH}$, et al. A novel classification system for spinal instability in neoplastic disease: an evidence-based approach and expert consensus from the Spine Oncology Study Group. Spine. 2010;35: E1221-E.

15. Fourney DR, Frangou EM, Ryken TC, DiPaola CP, Shaffrey CI, Berven SH, et al. Spinal instability neoplastic score: An analysis of reliability and validity from the spine Oncology Study Group. J Clin Oncol. 2011;29:3072-7.

16. Bollen L, Groenen K, Pondaag W, Van Rijswijk C, Fiocco M, Van der Linden Y, et al. Evaluation of the Spinal Instability Neoplastic Score for spinal metastases. Radiother Oncol. 2017;123: S74.

17. Tokuhashi Y, Matsuzaki H, Oda H, Oshima M, Ryu J. A revised scoring system for preoperative evaluation of metastatic spine tumor prognosis. Spine. 2005;30:2186-91.

18. Jensen G, Tang C, Hess KR, Bishop AJ, Pan HY, Li J, et al. Internal validation of the prognostic index for spine metastasis (PRISM) for stratifying survival in patients treated with spinal stereotactic radiosurgery. Int J Radiat Oncol. 2017;98: E15.

19. Rades D, Weber A, Karstens JH, Schild SE, Bartscht T. Prognostic role of the number of involved extraspinal organs in patients with metastatic spinal cord compression. Clin Neurol Neurosurg. 2014;118:12-5.

20. Mendel E, Bourekas EC, Porensky P. Vertebral augmentation for metastatic disease. In: Vialle LR, editor. AO Spine Masters Series Metastatic Spinal Tumors. Berlin: Thieme; 2015. P.8493.

21. Chen K, Huang L, Cai Z, Shi J, You K, Shen H. Micro-invasive surgery combined with intraoperative radiotherapy for the treatment of spinal metastasis. Eur Spine J. 2016;1-9.

22. Moulding HD, Elder JB, Lis E, Lovelock DM, Zhang Z, Yamada $Y$, et al. Local disease control after decompressive surgery and adjuvant high-dose single-fraction radiosurgery for spine metastases. J Neurosurg Spine. 2010;13:87-93.

23. Laufer I, Iorgulescu JB, Chapman T, Lis E, Shi W, Zhang Z, et al. Local disease control for spinal metastases following "separation surgery" and adjuvant hypofractionated or high-dose single-fraction stereotactic radiosurgery: outcome analysis in 186 patients. J Neurosurg Spine. 2013;18:207-14.

24. Laufer I, Wolinsky JP, Bilsky MH. En bloc resection in the treatment of spinal metastases: Technique and indications. In: Vialle LR, editor. AO Spine Masters Series Metastatic Spinal Tumors. Berlin: Thieme; 2015. p.34- 44. 